\title{
Interactive Non-Fiction: Towards a New Approach for Storytelling in Digital Journalism
}

\author{
J. Hunter Sizemore ${ }^{1}$ and Jichen $\mathrm{Zhu}^{2}$ \\ 1 School of Visual Arts and Design, \\ University of Central Florida, Orlando, FL 32826 \\ jhsizemore@knights.ucf.edu \\ 2 Antoinette Westphal College of Media Arts \& Design, \\ Drexel University, Philadelphia, PA 19104 \\ jichen.zhu@drexel.edu
}

\begin{abstract}
The development of digital journalism, an important area of storytelling, has focused primarily on incorporating interactive multimedia components to traditional linear news stories. In this paper, we propose a new approach that places central focus on text, the core of journalism, based on insights from fictional interactive digital storytelling. Drawing upon the convention of interactive fiction, we present our preliminary work on interactive non-fiction.
\end{abstract}

\section{Introduction}

Interactive digital storytelling (IDS) is a multi-disciplinary area that extends traditional storytelling techniques and conventions through digital media. So far, most efforts in IDS have been focused on fictional storytelling - imaginary story worlds that can take multiple shapes based on different parameters as a new mode of storytelling. In this paper, we explore the expressive affordance and potential issues of "multi-plot" storytelling in the setting of fact-based journalistic reporting.

It is true that digital technology has shaped journalism in multiple ways. However, a majority of such attempts focus on how to integrate multimedia content (e.g. images, animation, video) as a complement to text. The way we think about text, the core component of journalism, has remained fundamentally unchanged. In this paper, we propose a new way of integrating computation and journalism based on insights from fiction-based IDS systems. In particular, our current experiment draws from the convention of interactive fiction (IF), a genre primarily based on text. Our initial inquiry raises questions about the variability of text in the context of "journalistic truth," "balanced" reporting and reader agency. Our ultimate goal is to steer digital journalism from an "additive" formulation [6] into a more mature expressive form. In the rest of the paper, we present relevant background, introduce interactive non-fiction through a comparative reporting case study and conclude with discussions. 


\section{Background and Related Work}

Experiments utilizing digital technology for journalism purposes can be traced back to the early 1970s 3 . The prominent approach of contemporary digital journalism centers around various ways of incorporating multimedia into traditional reporting. For instance, McAdams's "Flash journalism" practice [5] calls for using multimedia platforms such as Adobe Flash to produce interactive, multimedia stories that enrich readers' experience and better explain often convoluted information through a collage of photos, sound, video, animation, infographics and text. By and large, text, the core of journalism, is still structured and consumed in ways resembling traditional newspaper [1].

The specific IDS tradition that we draw upon is interactive fiction (IF). Sometimes referred to as text adventures, IF is an interactive storytelling form that mixes elements from literary fiction and computer games, typically using text as its primary medium. A typical way to interact with an IF piece is through text commands. A user types in various commands, often in the form of verb-noun combination such as "pick up the key" or "go west." Through a text parser, the system analyzes the input and returns appropriate textual response. Depending on user interaction, each run-through of an IF system can yield different stories.

Although we are not aware of any IF-based interactive journalism practice, several simulation-based systems are related to our approach, such as Columbia University's News Simulation: A fire scenario project [2] and what Bogost et. al. call "news games" [1]. However, many existing projects emphasize editorial commentary rather than presenting neutral and balanced reporting and require high production cost. By comparison, our focus is on journalistic reporting and a scalable model that can be explored by many journalists in their daily practice.

\section{Interactive Non-Fiction}

Interactive non-fiction (INF) offers a different approach for rethinking the role of text in the context of digital journalism. We use the term to refer to text-based news stories presented using conventions borrowed from interactive fiction. A reader of INF interacts with the news story through an IF-like user interface, exploring viewpoints from different sources in the order and level of depth she desires. Whereas an IF piece may produce drastically different stories, INF is geared towards reflection of real-life events and requires multiple versions of the story to converge. This hence imposes a different set of constraints on how various story components can and should be composed together.

In our current work, we conducted a case study INF reporting and compared it with the equivalent story in the traditional form. Our source material comes from the comprehensive interview notes of a reporter who already published the story "Thermography Claims 'Misleading': FDA" in the traditional form on www.healthnewsflorida.org. The story covers a recently released Federal Drug 
>ask everyone about mammography

Carol Chandler, licensed acupuncture physician who sells thermograms:

"There are a lot of women who don't even have mammograms."

RELATED POINTS: CON TROVERSY OVER THERM OGRAPHY, SAFETY OF MAMMOGRAMS

Linda Brateman, physicist at the University of Florida:

"The probability of $\mathrm{x}$-ray mammography causing cancer is much less than the risk of not having a mammogram."

RELATED TOPICS: MAMM OGRAPHY AS A TOOL, MAMMOGRAPHY AS A

STAN DARD

>what is mammography

Mammography is the process of using low-dose amplitude-X-rays (usually around $0.7 \mathrm{mSv}$ ) to examine the human breast and is used as a diagnostic and a screening tool. The goal of mammography is the early detection of breast cancer, typically through detection of characteristic masses and/or microcalcifications. Mammography is believed to reduce mortality from breast cancer.

>ask chandler about mammography

\begin{abstract}
"There are a lot of women who don't even have mammograms. They just don't personally choose to do that. It's a woman's choice, and some woman choosenot to. Usually, one, for some women it's very painful-that's probably the number one reason women don't have it done. The other reason is radiation exposure," Chandler says.
\end{abstract}

Fig. 1. Screenshot of an Interactive Non-Fiction Reporting

Administration (FDA) warning against thermography as an alternative to mammography in breast cancer testing, reporting arguments against and for thermography. We chose this story because it contains a wide array of opinions on a controversial topic. Our initial design of an INF news story on the same subject is mocked up using the Inform 7 System, whose natural language-like programming language, multiple extensions, and an active support community offer a reasonable platform for journalists working under tight deadlines.

Unlike the established news structure in the original story, our INF reporting (Fig. 1 shows an excerpt) is framed with a format we call Round Table. Here, all available sources (interviewees) participate in a non-linear round table discussion with the reader on pre-defined topics. After the traditional "nutgraf," summarizing the news value of the story, the reader is presented with a list of sources: their names, titles and a small quote relating their positions on the main topic. There are three central navigation functions: "ask everyone [topic]," "what is [topic]," and "ask [NPC] about [topic]." The first function yields responses from all sources, each represented as a non-player character (NPC). Brief responses present each NPC's basic view on the subject. The second function supplements user's knowledge on basic subjects foundational to the story that may be taken for granted by sources in their interviews. The last function, which allows users to address specific NPCs, yields a source's extended opinion on a topic and, in some cases, elicit additional "related topics" recommending similar or opposing viewpoints from other sources. In addition, 
we also allow easier access to the main story for time-strapped readers. Several ways of doing this are the "list topic" command, which shows all of the topics in the story, the "what is [topic]" command, and the "glossary" command, which lists all of the key words related to the story.

A straightforward advantage of INF is that it allows a news story to be better tailored to individual readers. More important, it offers new opportunities for both news consumers and producers to engage news media more critically. Gilmore [4] argues that journalists often misrepresent the strength and popularity of minority opinions in an effort to achieve "balance." For example, in the case of global warming, one side is supported by substantial amount of scientific evidence while the opposition is small and presents little beyond their skepticism. Still, the majority of news stories mention the anti-global warming argument, fearing the criticism of cheerleading one side. Our proposal for INF starts to address the two-sides fallacy primarily by providing more levels of presentation beyond in/exclusion. A minority opinion, depending on its nature, may be accommodated in the main source responses, or as an optional branch that are multiple levels of interaction away from the main story.

\section{Conclusion}

In conclusion, our initial exploration of interactive non-fiction indicated that insights from fictional interactive narrative research are valuable for further transforming journalism in a more interactive and critical way. Through a comparative case study of reporting, we illustrated some of the differences between INF and traditional news stories as well as how INF may offer a model of journalistic balance that addresses the individual concerns of news consumers. For our future work, we plan to expand our Round Table concept by further exploiting IF's procedural nature such as the ability to model economic or political systems and track predefined NPC emotional responses. Levels of details of different viewpoints may also be modified procedurally based on the arguments that the user has already encountered.

\section{References}

[1] Bogost, I., Ferrari, S., Schweizer, B.: Newsgames: Journalism at Play. MIT Press, Cambridge (2010)

[2] Cameron, D.: Playing serious games in journalism classes. Asia Pacific Media Educator (11), 141-149 (2001)

[3] Carlson, D.: The history of online journalism. In: Kawamoto, K. (ed.) Digital Journalism: Emerging Media and the Changing Horizons of Journalism, pp. 31-56. Rowman \& Littlefield, Oxford (2003)

[4] Gillmor, D.: Mediactive. Dan Gillmor (2010)

[5] McAdams, M.: Flash Journalism: How to Create Multimedia News Packages. Focal Press, Amsterdam (2005)

[6] Murray, J.H.: Hamlet on the Holodeck: The Future of Narrative in Cyberspace. The MIT Press, Cambridge (1998) 